\title{
Optical Characterization of Electron-Phonon Interactions at the Saddle Point in Graphene
}

\author{
Adam T. Roberts, ${ }^{1,2}$ Rolf Binder, ${ }^{1,3}$ Nai H. Kwong, ${ }^{1}$ Dheeraj Golla, ${ }^{3}$ Daniel Cormode, ${ }^{3}$ Brian J. LeRoy, ${ }^{3}$ \\ Henry O. Everitt, ${ }^{2}$ and Arvinder Sandhu, ${ }^{1,3, *}$ \\ ${ }^{1}$ College of Optical Sciences, University of Arizona, Tucson, Arizona 85721, USA \\ ${ }^{2}$ U.S. Army Aviation and Missile Research, Development, and Engineering Center, Redstone Arsenal, Alabama 35898, USA \\ ${ }^{3}$ Department of Physics, University of Arizona, Tucson, Arizona 85721, USA
}

(Received 9 October 2013; published 8 May 2014)

\begin{abstract}
The role of many-body interactions is experimentally and theoretically investigated near the saddle point absorption peak of graphene. The time and energy-resolved differential optical transmission measurements reveal the dominant role played by electron-acoustic phonon coupling in band structure renormalization. Using a Born approximation for electron-phonon coupling and experimental estimates of the dynamic lattice temperature, we compute the differential transmission line shape. Comparing the numerical and experimental line shapes, we deduce the effective acoustic deformation potential to be $D_{\mathrm{eff}}^{\mathrm{ac}} \simeq 5 \mathrm{eV}$. This value is in accord with recent theoretical predictions but differs from those extracted using electrical transport measurements.
\end{abstract}

DOI: 10.1103/PhysRevLett.112.187401

PACS numbers: 78.67.Wj, 63.20.kd, 73.22.Pr, 78.47.J-

Graphene research is fueling the development of new electronic and photonic devices such as high-speed fieldeffect transistors, efficient terahertz sources, ultrafast broadband photodetectors, and photovoltaics [1,2]. Recent experimental and theoretical efforts have led to the recognition of the fact that electron-electron [3] and electron-phonon [4] couplings as well as excitonic effects [5-9] can significantly alter the electronic band structure and optical properties of graphene. When a light pulse interacts with graphene, the observation of many-body effects caused by transient photoexcited carriers is limited to short, subpicosecond time scales due to high electron scattering rates and short lifetimes. Over longer time scales (1-100 ps), the photoexcitation energy is converted into heat, and band structure renormalization effects due to electron-phonon interactions and possibly thermally excited charge carriers at the elevated temperatures [10] can be measured.

The long time scale electron-phonon ( $e$-ph) interactions in graphene have numerous ramifications. The intrinsic carrier mobility in high quality graphene devices is limited by $e$-ph scattering [11-15]. Efficient optoelectronic device design also relies on the conversion of the energy of photoexcited carriers to electrical current before it dissipates through $e$-ph interactions [16-18]. Ultrafast heat generation and dissipation dynamics in devices, which is an important topic in nanoscale heat management, is also crucially dependent on the interaction of electronic excitations with phonons [19].

The exact nature and strength of $e$-ph coupling in graphene is unclear at present. Specifically, the electronacoustic phonon interaction strength, characterized by the deformation potential $D_{\text {eff }}^{\text {ac }}$, has been controversial. The experimental estimates obtained from electrical transport measurements [13-15] range from 16-50 eV, while theoretical predictions indicate acoustic deformation potential in the range of $\sim 2.8-7 \mathrm{eV}[4,11,20,21]$. Since many observables are proportional to $\left|D_{\text {eff }}^{\text {ac }}\right|^{2}$, a $3-50 \mathrm{eV}$ range implies an uncertainty of more than two orders of magnitude. The optical phonon deformation potential $D_{\mathrm{eff}}^{\mathrm{op}}$ also show some variation; for example, Ref. [20] uses $11 \mathrm{eV} / \AA$, whereas Ref. [22] uses $50 \mathrm{eV} / \AA$.

Here we report ultrafast pump-probe measurements of photon energy dependent differential transmission in graphene. Instead of focusing on the heavily studied $K$ point of the graphene band structure [23-28], our study uniquely concentrates on the region near the $M$ point. The absorption maxima associated with the van Hove singularity at the $M$ point (Fig. 1) enables sensitive probing of $e$-ph interactions through the measurement of pump-induced changes in the absorption spectrum. In contrast, the region near the $K$ point has a relatively featureless, flat absorption profile which does not permit easy identification of many-body effects (Fig. 1). In this Letter, we investigate the complex line shape of differential transmission spectra obtained at various pump-probe time delays. For time scales $\geq 2 \mathrm{ps}$, we find that the renormalization predicted for $e$-ph interactions yields good qualitative and quantitative agreement with the measured spectra. In contrast, the renormalization predicted by statically screened Hartree-Fock calculations of electron-electron $(e-e)$ interactions among photoexcited or thermally excited carriers reproduces neither the shape nor the strength of the measured spectra. Furthermore, the Pauli blocking effect and the intraband absorption of photoexcited carriers were found to be insignificant. These findings allow us to estimate the acoustic 


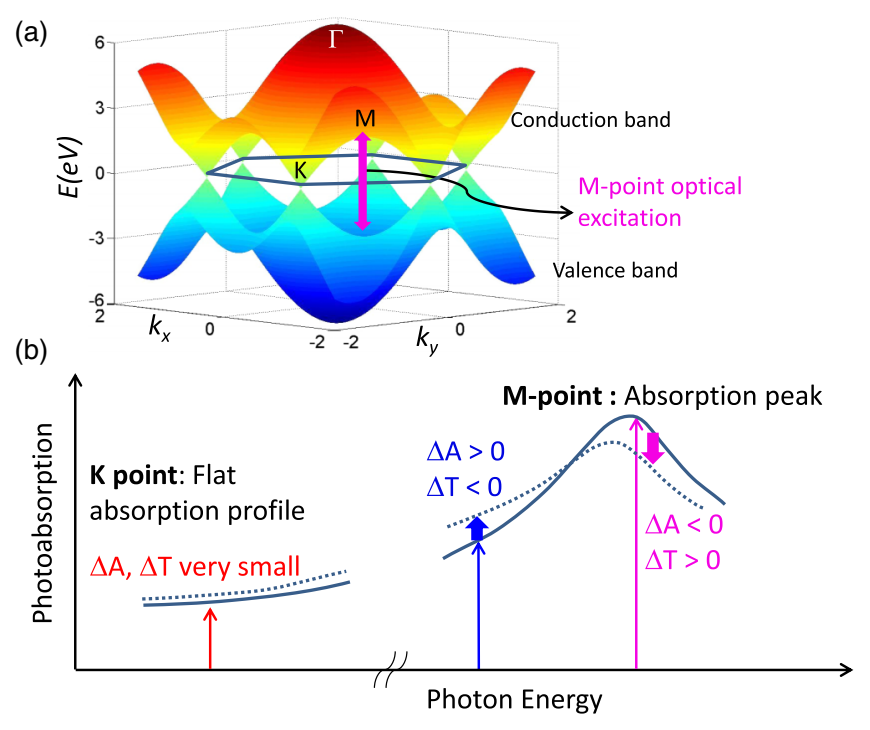

FIG. 1 (color online). (a) Electronic band structure of graphene. (b) Schematic variation of absorption with photon energy (solid curve). The $M$ point absorption peak corresponds to the saddle point in the band structure. We measure the pump-induced change in probe absorption (transmission) $\Delta A(\Delta T=1-\Delta A)$ which corresponds to the modification of the absorption profile (dashed curve) due to electron-phonon renormalization. Around the $M$ point, the energy-resolved differential transmission has a large magnitude and exhibits a sign change, enabling easy identification of many-body effects.

deformation potential to be $D_{\text {eff }}^{\mathrm{ac}} \simeq 5 \mathrm{eV}$, a value that is significantly lower than those obtained through transport measurements but quite consistent with recent theoretical predictions. Such optical measurements thus constitute a sensitive alternative to transport studies where the acoustic phonon contribution might be harder to differentiate in the presence of strong elastic-scattering and quantum resistance effects [17].

The graphene used in our study was grown through chemical vapor deposition [29] and then transferred to a $200 \mu \mathrm{m}$ thick sapphire substrate. In order to enhance the signal level in our measurements, up to ten individually grown graphene layers were stacked on top of each other. The layers in the stack do not influence the monolayer behavior of the sample as was verified through Raman spectroscopy. Prior linear absorption measurements [30] also indicate that there is minimal interlayer coupling in CVD graphene stacks compared to exfoliated samples or multilayer films on SiC. Additionally, we used Raman spectra to ascertain that our sample was slightly hole doped, with the Fermi level approximately at $-100 \mathrm{meV}$.

The time-resolved studies were performed using a $1 \mathrm{kHz}$ Ti:sapphire laser amplifier and an optical parametric amplifier. The pump and probe pulses of $\sim 100$ fs duration were focused slightly noncollinearly on the sample. The pump pulse photoexcites carriers, which relax through excitation of phonons [18]. The pump beam was chopped and lock-in amplification was used to detect changes in the transmission of the time delayed probe over a wide range of photon energies on either side of the $4.6 \mathrm{eV} M$ point resonance, which is a local "saddle-point" absorption maximum [5]. After the pump-produced carriers have substantially relaxed through phonon emission, the interaction of probe-excited electrons with the phonon population manifests itself as a renormalization of the band structure and modification of the probe absorption spectrum. Depending on the photon energy, the probe can thus experience a decrease or increase in the absorption (see Fig. 1), and the change in the sign of differential transmission provides unambiguous evidence of band renormalization due to $e$-ph interactions.

The transmission change measured in our experiment detects changes in the band structure induced by the pump pulse. If the pump does not raise the temperature to a value close to the optical phonon frequency $\hbar \Omega_{\text {opt }}(\simeq 200 \mathrm{meV})$, then the acoustic phonon interactions dominate the observed signal. As we discuss below, the highest estimated initial phonon temperature of $\mathcal{T}_{0}=610 \mathrm{~K}$ implies $k_{B} \mathcal{T} \simeq 53 \mathrm{meV}$, which is substantially smaller than $\hbar \Omega_{\text {opt }}$. This regime allows us to probe the electron-acoustic phonon coupling and distinguishes our work from angleresolved photoemission spectroscopy studies [31,32], which are more sensitive to optical phonon effects. Furthermore, in the context of optical studies of band renormalization, the phonon-dominated long time scale regime explored here is distinct from the previous studies which focused on excitonic effects [5,7-9] and short time scale carrier relaxation of photoexcited graphene [18,23-28,33-35].

The normalized pump-induced change in transmission $(\Delta T / T)$ of a ten monolayer graphene stack is shown in Fig. 2 as a function of the pump-probe time delay. In this figure, panels labeled (a), (b), and (c) correspond to the use of three different photon energies around the saddle point absorption maxima as shown in panel (d). Also, these measurements are degenerate in pump and probe energy (i.e., $\left.\hbar \omega_{\mathrm{pu}}=\hbar \omega_{\mathrm{pr}}\right)$. The pump fluence levels in Fig. 2 correspond to carrier densities $\sim 10^{12}-10^{13} \mathrm{~cm}^{-2}$. Figures 2(a) and 2(b) correspond to photon energies of 4.8 and $3.2 \mathrm{eV}$, respectively. Both figures show a positive transient lasting a few hundred femtoseconds, which can be attributed to Pauli blocking of the probe absorption by pump-induced electrons (holes) in the conduction (valence) band [23]. The carriers quickly relax through carrier-carrier and carrier-phonon scattering mechanisms [18,23-28, 33,34]. After 2 ps, the plots in Figs. 2(a) and 2(b) exhibit a slow relaxation to the zero baseline. Notably, the sign of $\Delta T / T$ at long time scales ( $>2 \mathrm{ps}$ ) is opposite in these two cases. For the $4.8 \mathrm{eV}$ case, we observe a strong positive $\Delta T / T$, while, for the $3.2 \mathrm{eV}$ case, it is negative. The negative $\Delta T / T$ indicates the generation of additional absorption channels which cannot be explained in the 


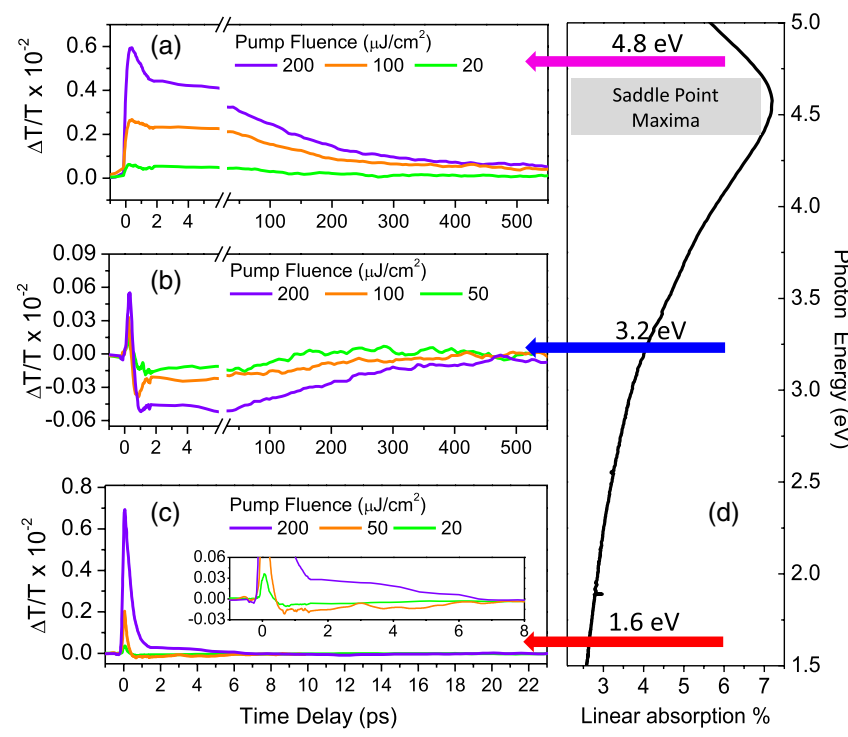

FIG. 2 (color online). $\Delta T / T$ of the probe pulse at pump-probe energies of (a) $4.8 \mathrm{eV}$, (b) $3.2 \mathrm{eV}$, and (c) $1.6 \mathrm{eV}$. In each case different curves correspond to different pump fluence levels. (d) The linear absorption profile indicating the probing energies relative to the saddle point absorption maxima.

independent particle picture. The magnitude of the $\Delta T / T$ change is found to be proportional to the pump fluence (i.e., carrier density) in both cases. Importantly, the $\Delta T / T$ change lasts for the same duration in both cases $(\sim 300 \mathrm{ps})$, which suggests a common origin despite opposite signs. As discussed below, these observations are due to the $e$-ph interaction induced band renormalization.

It is useful to compare 3.2 and $4.8 \mathrm{eV}$ observations with the often studied case of $\hbar \omega_{\mathrm{pu}}=\hbar \omega_{\mathrm{pr}}=1.6 \mathrm{eV}$. Our $1.6 \mathrm{eV}$ data [Fig. 2(c)] show a positive transient followed by a decay within $1 \mathrm{ps}$, and a very small $\Delta T / T$ change beyond 2 ps time delay. These observations are consistent with Pauli-blocking and carrier relaxation mechanisms discussed extensively in the literature [23,25-27] and indicate that substantial phonon excitation and carrier recombination occurs within the first 2 ps. The transient signals beyond 2 ps are thus expected to be dominated by phonon-induced renormalization. However, as shown in the zoomed in view [inset, Fig. 2(c)], the positive or negative transients in the $1.6 \mathrm{eV}$ data are extremely weak beyond 2 ps. Therefore, near-infrared $(\sim 1.6 \mathrm{eV})$ measurements in the Dirac cone region, where the linear absorption profile is essentially flat, do not permit clear identification of the $e$-ph interactions. In contrast, observations at higher photon energies exhibit stronger transients at long time delays, where $e$-ph interactions dominate.

Specifically, the energy resolved measurements around the saddle point absorption maxima can serve to quantify $e$-ph coupling effects through large changes in the magnitude and accompanied by changes in the sign of $\Delta T / T$. In fact, hints of phonon induced band renormalization have been observed in some previous studies at high photon energies [34,36]. A detailed measurement of the "line shape," i.e., the energy dependence of $\Delta T / T$ near the saddle point energy at time delays greater than $2 \mathrm{ps}$, can thus provide a means to quantify the strength of $e$-ph interactions. We obtain the experimental line shape of $\Delta T / T$, by fixing the pump excitation energy to $4.8 \mathrm{eV}$ and varying the probe photon energy around the saddle point transition. These measurements are shown in Fig. 3 for time delays of 4,40 , and $400 \mathrm{ps}$. The fact that the line shape is similar for all three delay times suggests that the microscopic process giving rise to renormalization is the same from 4 to $400 \mathrm{ps}$. Since $e$ - $e$ interactions cannot dominate at long time delay $(\sim 400 \mathrm{ps})$ due to established carrier relaxation rates, $e$-ph interactions form a strong candidate for explaining our observations.

The band structure renormalization is a correction to the electron energy $E_{s}(\mathbf{k})$ (where $\mathbf{k}$ is the electron wave vector
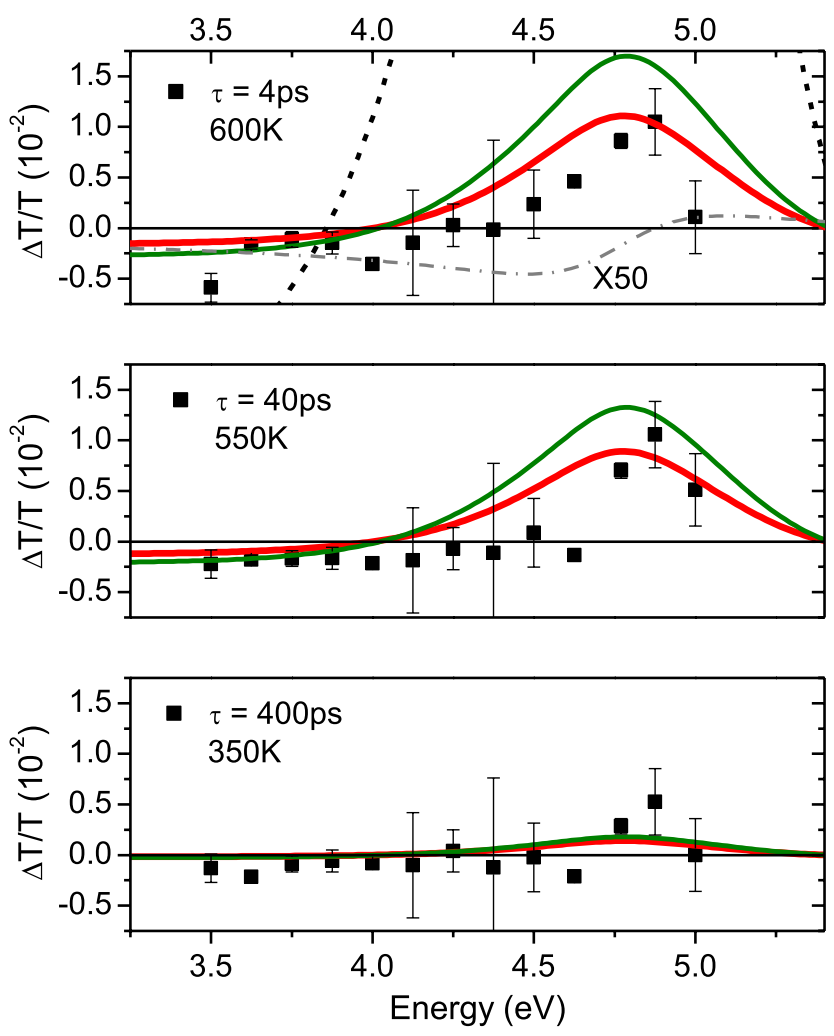

FIG. 3 (color online). $\Delta T / T$ as a function of probe photon energy at three time delays (4, 40, and $400 \mathrm{ps}$ ). The pump pulse fluence is $400 \mu \mathrm{J} / \mathrm{cm}^{2}$ and fixed excitation energy of $4.8 \mathrm{eV}$. Each panel is labeled with a specific phonon temperature value. The calculated results for coupling to acoustic phonons only with $D_{\text {eff }}^{\text {ac }}=5.3 \mathrm{eV}$ are shown in red (thick line) and acoustic + optical phonons with $D_{\text {eff }}^{\text {op }}=11 \mathrm{eV} / \AA$ are shown in green (thin line). The dashed black line (top panel) shows acoustic-only coupling with $D_{\text {eff }}^{\text {ac }}=20 \mathrm{eV}$. The dash-dotted gray line (top panel) shows the statically screened Hartree-Fock result for $e-e$ interaction (multiplied by 50 for ease of comparison) using thermally excited carriers. 
and $s= \pm 1$ is the band index) due to self-energy terms $\Sigma_{s}(\mathbf{k})$ associated with $e$-ph and $e-e$ interactions. To identify the exact nature of many-body effects manifested in our $\Delta T / T$ measurements, we calculate changes in $e$-ph and/or $e$-e self-energies $\Delta \Sigma_{s}(\mathbf{k})$ induced by the pump pulse [37]. $\Delta \Sigma_{s}(\mathbf{k})$ depends on the pump-probe delay via the timedependent phonon occupation functions and Fermi distributions (for example, through temperature increase). Using the time-dependent $\Delta \Sigma_{s}(\mathbf{k})$ values, we numerically compute the susceptibility to obtain the time and energy resolved $\Delta T / T$ profiles [37]. For $e$-ph coupling, the self-energy depends on the matrix element

$$
\left|M_{s, s^{\prime}}^{\mathrm{ac}}\left(\mathbf{k}, \mathbf{k}^{\prime}\right)\right|^{2}=\frac{\hbar \mathcal{A}_{o}}{4 m \omega_{\mathbf{k}-\mathbf{k}^{\prime}}^{\mathrm{LA}}}\left|D_{\mathrm{eff}}^{\mathrm{ac}} q\left\langle u_{s \mathbf{k}} \mid u_{s^{\prime} \mathbf{k}^{\prime}}\right\rangle\right|^{2},
$$

where the longitudinal acoustic phonon frequencies are denoted by $\omega_{\mathbf{q}}^{\mathrm{LA}}$ (all phonon frequencies are obtained numerically after Ref. [38]), $m$ is the carbon nucleus mass, $\mathcal{A}_{0}$ the area of the unit cell, and $\left|u_{\mathbf{k}^{\prime}, s}\right\rangle$ are the latticeperiodic parts of the two-component spinor Bloch function. An effective deformation potential $D_{\text {eff }}^{\text {ac }}$ with coupling to longitudinal phonons (denoted by $\Xi_{\text {eff in Ref. [4]) has been }}$ found to provide a quantitative model for the combined coupling to longitudinal and transverse acoustic phonons. Our model considers only the coupling of electrons to inplane phonon modes because out-of-plane phonon modes are expected to have a negligible contribution [11,39]. For coupling to optical phonons, we proceed in the same semiphenomenological manner [37].

To compare the theoretical calculation of differential transmission line shape with the observed experimental data, the knowledge of phonon temperature $\mathcal{T}(\tau)$ at various time delays is required. We estimate the initial temperature $\mathcal{T}_{0}$ by assuming that within a short time $\tau<2$ ps [18,23,25-27,34], the photoexcited carriers relax and the supplied laser pulse energy $E_{L}$ is transferred to the phonons with internal energy given by

$$
U_{\mathrm{ph}}\left(\mathcal{T}_{0}\right)=\sum_{\mathbf{q} \mu} \hbar \omega_{\mathbf{q}}^{\mu}\left[n_{\mu}^{\mathrm{ph}}\left(\mathbf{q}, \mathcal{T}_{0}\right)+1 / 2\right]
$$

where $\mu$ sums over all in-plane phonon branches, and $n_{\mu}^{\mathrm{ph}}$ denotes the Bose phonon occupation function. By calculating the internal energy of phonons as a function of temperature, the initial temperature is found using $U_{\mathrm{ph}}\left(\mathcal{T}_{0}\right)=U_{\mathrm{ph}}(300 \mathrm{~K})+E_{L}$. For the highest incident fluence of $400 \mu \mathrm{J} / \mathrm{cm}^{2}$, the estimated initial temperature of the phonon system is $\mathcal{T}_{0}=610 \mathrm{~K}$. The temperature will decrease as the phonons decay and transfer energy to the substrate. We find that in the $e$-ph coupling dominated region ( $\tau>2 \mathrm{ps}$ ), the $\Delta T / T$ at $4.8 \mathrm{eV}$ is linearly related to the temperature, so the temperature decay profile may be estimated by fitting an exponential decay curve to $\Delta T / T$ at $4.8 \mathrm{eV}$ in Fig. 2(a).
Using this method, the phonon temperatures $\mathcal{T}(\tau)$ are estimated to be 600,550 , and $350 \mathrm{~K}$ at time delays of $\tau=4$, 40 , and $400 \mathrm{ps,} \mathrm{respectively.} \mathrm{The} \mathrm{numerical} \mathrm{calculations}$ using only electron-acoustic phonon coupling and the predicted value of $D_{\text {eff }}^{\text {ac }} \simeq 5 \mathrm{eV}[4,40]$ reproduce the measured line shape, the $\Delta T / T$ maxima, and the zero-point crossing quite well for all three time delays (Fig. 3, red curves). Both the real (band shift) and imaginary (line broadening) part of self-energy change contribute to the signal, with values near the $M$ point of about 3 and $6 \mathrm{meV}$, respectively, at $600 \mathrm{~K}$. Note that $D_{\text {eff }}^{\text {ac }}=20 \mathrm{eV}$, a value typically reported in transport studies, yields an inordinately large $\Delta T / T$ (dashed line, Fig. 3 top panel) inconsistent with our measurements.

Although there is overall good agreement with the data using the theoretically predicted value of $D_{\text {eff }}^{\text {ac }}$, further refinements are possible. Adding the optical phonon contribution increases the signal slightly but leaves the line shape unchanged. Using $D_{\mathrm{eff}}^{\mathrm{op}}=11 \mathrm{eV} / \AA$ from Ref. [20] still yields reasonable agreement with experiment, especially at 40 and $400 \mathrm{ps}$ (Fig. 3, green curves). Note that the calculated zero-crossing energy of $4.1 \mathrm{eV}$ differs slightly from the experimentally measured value of $4.3 \mathrm{eV}$ (Fig. 3). Since the zero-crossing point is an important and stable feature of the experimental line shape, it could be used as a guide for further extensions and improvements to the theory. For example, microscopic modeling of excitonic effects in the probe absorption and in the $e$-ph coupling might further reduce the small discrepancies between theory and experiment. Furthermore, understanding a possible dependence of $D_{\text {eff }}$ on the substrate may help to reduce discrepancies in the overall size of the observed and calculated signal.

While the renormalization due to $e$-ph coupling explains the long time scale measurements well, we can still estimate the contribution from $e$-e interactions. Our simulations show that Pauli blocking is insignificant after 2 ps, when the system is in a quasithermal equilibrium. Using the results in [10] we estimate that free carrier intraband absorption at our photon energies can only produce a few percent correction. To show that $e$ - $e$ interactions are not significant on our time scale, we followed the strategy of [10] to estimate the carrier density difference between 300 and $600 \mathrm{~K}$ and computed the corresponding renormalization difference. Using the statically screened HartreeFock approximation [37], we find that the magnitude of the computed signal (dash-dotted curve, Fig. 3 top panel) is very small and the computed line shape is very different from our observations.

In summary, we present differential transmission spectra of graphene over a range of frequencies in the vicinity of the $M$ point. Our work demonstrates that differential transmission line shape measurements can be used for identifying the nature of many-body interactions. The observed line shape is consistent with a many-particle 
theory using an effective electron-acoustic-phonon deformation potential of $D_{\mathrm{eff}}^{\mathrm{ac}} \simeq 5 \mathrm{eV}$. Future time-resolved studies using nonlinear spectroscopy at the $M$ point could investigate additional physical processes such as manybody effects associated with fast carrier dynamics occurring during the initial femtosecond time scales and the role of excitonic-phonon coupling.

We thank J. V. Foreman for assistance with experiments. A. R. acknowledges support from the DoD SMART program. D. C. and B. J. L. acknowledge support from the U.S. Army Research Laboratory Grant No. W911NF-09-1-0333 and NSF Grant No. EECS-0925152.

*sandhu@physics.arizona.edu

[1] K. S. Novoselov, V. I. Fal'ko, L. Colombo, P. R. Gellert, M. G. Schwab, and K. Kim, Nature (London) 490, 192 (2012).

[2] F. Bonaccorso, Z. Sun, T. Hasan, and A. C. Ferrari, Nat. Photonics 4, 611 (2010).

[3] V. N. Kotov, B. Uchoa, V. M. Pereira, F. Guinea, and A. H. C. Neto, Rev. Mod. Phys. 84, 1067 (2012).

[4] K. Kaasbjerg, K. S. Thygesen, and K. W. Jacobsen, Phys. Rev. B 85, 165440 (2012).

[5] K. F. Mak, J. Shan, and T. F. Heinz, Phys. Rev. Lett. 106, 046401 (2011).

[6] D.-H. Chae, T. Utikal, S. Weisenburger, H. Giessen, K. v. Klitzing, M. Lippitz, and J. Smet, Nano Lett. 11, 1379 (2011).

[7] L. Yang, J. Deslippe, C. H. Park, M. L. Cohen, and S. G. Louie, Phys. Rev. Lett. 103, 186802 (2009).

[8] E. Malic, T. Winzer, E. Bobkin, and A. Knorr, Phys. Rev. B 84, 205406 (2011).

[9] J. Grönqvist, T. Stroucken, M. Lindberg, and S. Koch, Eur. Phys. J. B 85, 395 (2012).

[10] L. Malard, K. Mak, A. Castro Neto, N. Peres, and T. Heinz, New J. Phys. 15, 015009 (2013).

[11] K. M. Borysenko, J. T. Mullen, E. A. Barry, S. Paul, Y. G. Semenov, J. M. Zavada, M. B. Nardelli, and K. W. Kim, Phys. Rev. B 81, 121412 (2010).

[12] H. K. Min, E. H. Hwang, and S. Das Sarma, Phys. Rev. B 83, 161404 (2011).

[13] K. I. Bolotin, K. J. Sikes, J. Hone, H. L. Stormer, and P. Kim, Phys. Rev. Lett. 101, 096802 (2008).

[14] J. H. Chen, C. Jang, S. D. Xiao, M. Ishigami, and M. S. Fuhrer, Nat. Nanotechnol. 3, 206 (2008).

[15] C. R. Dean, A. F. Young, I. Meric, C. Lee, L. Wang, S. Sorgenfrei, K. Watanabe, T. Taniguchi, P. Kim, K. L. Shepard, and J. Hone, Nat. Nanotechnol. 5, 722 (2010).

[16] W. K. Tse and S. Das Sarma, Phys. Rev. B 79, 235406 (2009).

[17] R. Bistritzer and A. H. MacDonald, Phys. Rev. Lett. 102, 206410 (2009).

[18] E. Malic, T. Winzer, E. Bobkin, and A. Knorr, Phys. Rev. B 84, 205406 (2011).

[19] E. Pop, Nano Res. 3, 147 (2010).
[20] T. Low, V. Perebeinos, R. Kim, M. Freitag, and P. Avouris, Phys. Rev. B 86, 045413 (2012).

[21] V. Perebeinos and P. Avouris, Phys. Rev. B 81, 195442 (2010).

[22] N. Sule and I. Knezevic, J. Appl. Phys. 112, 053702 (2012).

[23] M. Breusing, S. Kuehn, T. Winzer, E. Malic, F. Milde, N. Severin, J. P. Rabe, C. Ropers, A. Knorr, and T. Elsaesser, Phys. Rev. B 83, 153410 (2011).

[24] S. Winnerl, M. Orlita, P. Plochocka, P. Kossacki, M. Potemski, T. Winzer, E. Malic, A. Knorr, M. Sprinkle, C. Berger, W. A. de Heer, H. Schneider, and M. Helm, Phys. Rev. Lett. 107, 237401 (2011).

[25] D. Sun, C. Divin, C. Berger, W. A. de Heer, P. N. First, and T. B. Norris, Phys. Status Solidi C 8, 1194 (2011).

[26] R. W. Newson, J. Dean, B. Schmidt, and H. M. van Driel, Opt. Express 17, 2326 (2009).

[27] J. M. Dawlaty, S. Shivaraman, M. Chandrashekhar, F. Rana, and M. G. Spencer, Appl. Phys. Lett. 92, 042116 (2008).

[28] D. Sun, Z. K. Wu, C. Divin, X. B. Li, C. Berger, W. A. de Heer, P. N. First, and T. B. Norris, Phys. Rev. Lett. 101, 157402 (2008).

[29] X. S. Li, W. W. Cai, J. H. An, S. Kim, J. Nah, D. X. Yang, R. Piner, A. Velamakanni, I. Jung, E. Tutuc, S. K. Banerjee, L. Colombo, and R. S. Ruoff, Science 324, 1312 (2009).

[30] T. Yu, J. Z. Shang, J. Y. Lin, and G. G. Gurzadyan, ACS Nano 5, 3278 (2011).

[31] A. Bostwick, T. Ohta, T. Seyller, K. Horn, and E. Rotenberg, Nat. Phys. 3, 36 (2007).

[32] D. A. Siegel, C. H. Park, C. Hwang, J. Deslippe, A. V. Fedorov, S. G. Louie, and A. Lanzara, Proc. Natl. Acad. Sci. U.S.A. 108, 11365 (2011).

[33] J. H. Strait, H. N. Wang, S. Shivaraman, V. Shields, M. Spencer, and F. Rana, Nano Lett. 11, 4902 (2011).

[34] J. Z. Shang, Z. Q. Luo, C. X. Cong, J. Y. Lin, T. Yu, and G. G. Gurzadyan, Appl. Phys. Lett. 97, 163103 (2010).

[35] P. Plochocka, P. Kossacki, A. Golnik, T. Kazimierczuk, C. Berger, W. A. de Heer, and M. Potemski, Phys. Rev. B 80, 245415 (2009).

[36] K. Seibert, G. C. Cho, W. Kutt, H. Kurz, D. H. Reitze, J. I. Dadap, H. Ahn, M. C. Downer, and A. M. Malvezzi, Phys. Rev. B 42, 2842 (1990).

[37] See Supplemental Material at http://link.aps.org/ supplemental/10.1103/PhysRevLett.112.187401 for details of theoretical calculations.

[38] H. Suzuura and T. Ando, Phys. Rev. B 65, 235412 (2002).

[39] E. Mariani and F. von Oppen, Phys. Rev. Lett. 100, 076801 (2008).

[40] Although our measurement is at the $M$ point, we expect $D_{\text {eff }}^{\text {ac }}(K) \simeq D_{\text {eff }}^{\text {ac }}(M)$. The acoustic deformation potential can be written as the area $(A)$ derivative of the electronic band energies as, $D_{s}^{\mathrm{ac}}(\mathbf{k}) \simeq D_{0}+A \partial E_{s}(\mathbf{k}) / \partial A$ [41]. Even though the concept of acoustic deformation potentials is usually restricted to band extrema, it is instructive to evaluate $D_{s}^{\mathrm{ac}}(\mathbf{k})$. We find that, on the $K-M$ line, $A \partial E_{s}(\mathbf{k}) / \partial A$ exhibits only a relatively moderate variation between -3 and $-5 \mathrm{eV}$.

[41] J. A. Verges, D. Glötzel, M. Cardona, and O. K. Andersen, Phys. Status Solidi (b) 113, 519 (1982). 